Georgian Mathematical Journal

Volume 13 (2006), Number 4, 793-805

\title{
ON A DOUBLE SERIES OF CHAN AND ONG
}

\author{
KENNETH S. WILLIAMS
}

\begin{abstract}
An arithmetic identity is used to prove a relation satisfied by the double series $\sum_{m, n=-\infty}^{\infty} q^{m^{2}+m n+2 n^{2}}$. As an application an explicit formula is given for the number of representations of the positive integer $n$ by the form $x_{1}^{2}+x_{1} x_{2}+2 x_{2}^{2}+x_{3}^{2}+x_{3} x_{4}+2 x_{4}^{2}+x_{5}^{2}+x_{5} x_{6}+2 x_{6}^{2}+x_{7}^{2}+x_{7} x_{8}+2 x_{8}^{2}$.
\end{abstract}

2000 Mathematics Subject Classification: 11F27, 11E25.

Key words and phrases: Eisenstein series, theta series, quadratic forms in 4 and 8 variables.

1. Introduction. Let $\mathbb{N}, \mathbb{N}_{0}, \mathbb{Z}, \mathbb{R}, \mathbb{C}$ denote the sets of positive integers, nonnegative integers, integers, real numbers, complex numbers, respectively. For $m \in \mathbb{Z}$ and $n \in \mathbb{N}$ we define

$$
\sigma_{m}(n):=\sum_{\substack{d \in \mathbb{N} \\ d \mid n}} d^{m}
$$

where $d$ runs through the positive integers dividing $n$. We also set $\sigma(n)=$ $\sigma_{1}(n)=\sum_{d \mid n} d$ and $d(n)=\sigma_{0}(n)=\sum_{d \mid n} 1$. If $n \notin \mathbb{N}$, we set $\sigma_{m}(n)=0$. The Bernoulli numbers $B_{0}=1, B_{1}=-\frac{1}{2}, B_{2}=\frac{1}{6}, B_{3}=0, B_{4}=-\frac{1}{30}, \ldots$ are defined by

$$
\frac{x}{e^{x}-1}=\sum_{n=0}^{\infty} B_{n} \frac{x^{n}}{n !}, \quad x \in \mathbb{R}, \quad|x|<2 \pi .
$$

The Eisenstein series $E_{k}(q)(k \in \mathbb{N})$ is defined by

$$
E_{k}(q):=1-\frac{4 k}{B_{2 k}} \sum_{n=1}^{\infty} \sigma_{2 k-1}(n) q^{n}, \quad q \in \mathbb{C}, \quad|q|<1 .
$$

We set

$$
L(q):=E_{1}(q)=1-24 \sum_{n=1}^{\infty} \sigma(n) q^{n} .
$$

In this paper we use a recent elementary arithmetic identity due to Huard, $\mathrm{Ou}$, Spearman and Williams [3] to prove in Section 5 the following result, after some preliminary results are proved in Sections 2, 3 and 4.

Theorem 1.1. Let $n \in \mathbb{N}$. Set $n=7^{\alpha} N$, where $\alpha \in \mathbb{N}_{0}, N \in \mathbb{N}$ and $\operatorname{gcd}(N, 7)=1$. Then the number of $(x, y, z, t) \in \mathbb{Z}^{4}$ such that

$$
n=x^{2}+x y+2 y^{2}+z^{2}+z t+2 t^{2}
$$


$i s$

$$
4 \sigma(n)-28 \sigma\left(\frac{n}{7}\right)=4 \sigma(N)=4 \sum_{\substack{d \mid n \\ 7 \nmid d}} d
$$

In 1999 H. H. Chan and Y. L. Ong [2] introduced the two-dimensional theta series

$$
S(q):=\sum_{m, n=-\infty}^{\infty} q^{m^{2}+m n+2 n^{2}}, \quad q \in \mathbb{C}, \quad|q|<1 .
$$

They proved a result equivalent to the following identity [2, Remark 3, p. 1742].

Theorem 1.2. $S^{2}(q)=\frac{7}{6} L\left(q^{7}\right)-\frac{1}{6} L(q)$.

This identity is also equivalent to the one stated by Ramanujan as entry 5 of his second notebook [10] and first proved by Berndt [1, p. 467, entry 5(i)]. Both Berndt and Chan and Ong used modular equations of degree 7 in their proofs of Theorem 1.2. We show in Section 6 that Theorem 1.2 is a simple consequence of Theorem 1.1 and thus can be viewed as an elementary identity.

Klein and Fricke in their book [6, p. 400] gave an analytic proof of the following theorem.

Theorem 1.3. Let $n \in \mathbb{N}$. Then the number of $(x, y, z, t) \in \mathbb{Z}^{4}$ such that

$$
4 n=x^{2}+y^{2}+7 z^{2}+7 t^{2}, \quad x \equiv z(\bmod 2)
$$

$i s$

$$
4 \sum_{\substack{d \mid n \\ 7 \nmid d}} d
$$

We show in Section 7 that Theorem 1.3 is also an elementary consequence of Theorem 1.1, thus providing an elementary proof of Theorem 1.3. The elementary proof of Theorem 1.3 given by Humbert [4] is restricted to odd $n$.

Next, making use of a result, which was proved recently by Lemire and Williams [8, Lemma 4.6, p. 113] in order to evaluate the convolution sum

$$
\sum_{\substack{m \in \mathbb{N} \\ 1 \leq m<\frac{n}{7}}} \sigma(m) \sigma(n-7 m),
$$

in conjunction with Theorem 1.2, we prove in Section 8 the following result.

Theorem 1.4. Let $n \in \mathbb{N}$. Then the number of $\left(x_{1}, x_{2}, x_{3}, x_{4}, x_{5}, x_{6}, x_{7}, x_{8}\right) \in$ $\mathbb{Z}^{8}$ such that

$$
n=x_{1}^{2}+x_{1} x_{2}+2 x_{2}^{2}+x_{3}^{2}+x_{3} x_{4}+2 x_{4}^{2}+x_{5}^{2}+x_{5} x_{6}+2 x_{6}^{2}+x_{7}^{2}+x_{7} x_{8}+2 x_{8}^{2}
$$

is given by

$$
\frac{24}{5} \sigma_{3}(n)+\frac{1176}{5} \sigma_{3}\left(\frac{n}{7}\right)+\frac{16}{5} c_{7}(n)
$$


where the $c_{7}(n)(n \in \mathbb{N})$ are integers defined by

$$
\begin{aligned}
\sum_{n=1}^{\infty} c_{7}(n) q^{n}=q & \left(\prod_{n=1}^{\infty}\left(1-q^{n}\right)^{16}\left(1-q^{7 n}\right)^{8}+13 q \prod_{n=1}^{\infty}\left(1-q^{n}\right)^{12}\left(1-q^{7 n}\right)^{12}\right. \\
& \left.+49 q^{2} \prod_{n=1}^{\infty}\left(1-q^{n}\right)^{8}\left(1-q^{7 n}\right)^{16}\right)^{\frac{1}{3}}
\end{aligned}
$$

This result should be compared with that of Kachakhidze [5].

Finally, we make use of a classical identity of Jacobi, which is given for example in [7, Corollary 6, p. 37], to prove the following formula for $c_{7}(n)$ $(n \in \mathbb{N})$ in Section 9 .

Theorem 1.5. For $n \in \mathbb{N}$ we have

$$
\begin{gathered}
c_{7}(n)=\sum_{\substack{(r, s) \in \mathbb{N}_{0}^{2} \\
\frac{r(r+1)}{2}+7 \frac{s(s+1)}{2}=n-1}}(-1)^{r+s}(2 r+1)(2 s+1) \\
+2 \sum_{\substack{(r, s, t) \in \mathbb{N}_{0}^{2} \times \mathbb{N} \\
\frac{r(r+1)}{2}+7 \frac{s(s+1)}{2}+t=n-1}}(-1)^{r+s}(2 r+1)(2 s+1) \sum_{\substack{d \in \mathbb{N} \\
d \mid t}}\left(\frac{-7}{d}\right) .
\end{gathered}
$$

Here

$$
\left(\frac{-7}{d}\right)= \begin{cases}1, & \text { if } d \equiv 1,2,4(\bmod 7) \\ -1, & \text { if } d \equiv 3,5,6(\bmod 7) \\ 0, & \text { if } d \equiv 0(\bmod 7)\end{cases}
$$

is the Legendre-Jacobi-Kronecker symbol for discriminant -7 .

2. Some properties of $F_{k}(n)$. For $k \in \mathbb{N}$ and $n \in \mathbb{Z}$ we define

$$
F_{k}(n):= \begin{cases}1, & \text { if } k \mid n, \\ 0, & \text { if } k \nmid n .\end{cases}
$$

Let $a \in \mathbb{Z}$. Denote the gcd of $k$ and $a$ by $(k, a)$. Clearly

$$
F_{k}(a n)=F_{k /(k, a)}(n) .
$$

For $x \in \mathbb{R}$ we denote the greatest integer less than or equal to $x$ by $[x]$. The following results are easily proved:

$$
\begin{aligned}
\sum_{d \mid n} F_{k}(d) & =d\left(\frac{n}{k}\right) ; \\
\sum_{d \mid n} d F_{k}(d) & =k \sigma\left(\frac{n}{k}\right) ; \\
\sum_{d \mid n} \frac{n}{d} F_{k}(d) & =\sigma\left(\frac{n}{k}\right) ;
\end{aligned}
$$




$$
\begin{aligned}
& \sum_{1 \leq l \leq m} F_{k}(l)=\left[\frac{m}{k}\right] \\
& \sum_{\substack{1 \leq l \leq m \\
2 \mid l}} F_{k}(l)= \begin{cases}{\left[\frac{m}{k}\right],} & \text { if } 2 \mid k, \\
{\left[\frac{m}{2 k}\right],} & \text { if } 2 \nmid k ;\end{cases} \\
& \sum_{\substack{1 \leq l \leq m \\
2 \nmid l}} F_{k}(l)= \begin{cases}0, & \text { if } 2 \mid k, \\
{\left[\frac{m+k}{2 k}\right],} & \text { if } 2 \nmid k ;\end{cases} \\
& \sum_{\substack{d|n \\
2| d}} \sum_{\substack{1 \leq l \leq d \\
2 \mid l}} F_{k}(l)= \begin{cases}\sum_{d \mid \frac{n}{2}}\left[\frac{2 d}{k}\right], & \text { if } 2 \mid k, \\
\sum_{d \mid \frac{n}{2}}\left[\frac{d}{k}\right], & \text { if } 2 \nmid k ;\end{cases} \\
& \sum_{\substack{d|n \\
2| d}} \sum_{\substack{1 \leq l \leq d \\
2 \nmid l}} F_{k}(l)= \begin{cases}0, & \text { if } 2 \mid k, \\
\sum_{d \mid \frac{n}{2}}\left[\frac{2 d+k}{2 k}\right], & \text { if } 2 \nmid k ;\end{cases} \\
& \sum_{\substack{d \mid n \\
2 \nmid d}} \sum_{\substack{1 \leq l \leq d \\
2 \mid l}} F_{k}(l)= \begin{cases}\sum_{d \mid n}\left[\frac{d}{k}\right]-\sum_{d \mid \frac{n}{2}}\left[\frac{2 d}{k}\right], & \text { if } 2 \mid k, \\
\sum_{d \mid n}\left[\frac{d}{2 k}\right]-\sum_{d \mid \frac{n}{2}}\left[\frac{d}{k}\right], \quad \text { if } 2 \nmid k ;\end{cases} \\
& \sum_{\substack{d \mid n \\
2 \nmid d}} \sum_{\substack{1 \leq l \leq d \\
2 \nmid l}} F_{k}(l)=\left\{\sum_{d \mid n}^{0,}\left[\frac{d+k}{2 k}\right]-\sum_{d \mid \frac{n}{2}}\left[\frac{2 d+k}{2 k}\right], \quad \text { if } 2 \nmid k .\right.
\end{aligned}
$$

Adding (2.9) and (2.12) we obtain

$$
\begin{aligned}
\sum_{d \mid n} \sum_{\substack{1 \leq l \leq d \\
l \equiv d(\bmod 2)}} F_{k}(l) & \\
& = \begin{cases}\sum_{d \mid n / 2}\left[\frac{2 d}{k}\right], & \text { if } 2 \mid k, \\
\sum_{d \mid n / 2}\left[\frac{d}{k}\right]+\sum_{d \mid n}\left[\frac{d+k}{2 k}\right]-\sum_{d \mid n / 2}\left[\frac{2 d+k}{2 k}\right], & \text { if } 2 \nmid k .\end{cases}
\end{aligned}
$$

3. An identity of Huard, Ou, Spearman and Williams. Using nothing more than the rearrangement of terms in finite sums, Huard, Ou, Spearman and Williams [3] proved the following elementary arithmetic formula. 
Theorem 3.1. Let $F: \mathbb{Z}^{4} \rightarrow \mathbb{C}$ be such that

$$
F(a, b, x, y)-F(x, y, a, b)=F(-a,-b, x, y)-F(x, y,-a,-b)
$$

for all $(a, b, x, y) \in \mathbb{Z}^{4}$. Then, for $n \in \mathbb{N}$, we have

$$
\begin{aligned}
& \sum_{\substack{(a, b, x, y) \in \mathbb{N}^{4} \\
a x+b y=n}}(F(a, b, x,-y)-F(a,-b, x, y)+F(a, a-b, x+y, y) \\
- & F(a, a+b, y-x, y)+F(b-a, b, x, x+y)-F(a+b, b, x, x-y)) \\
= & \sum_{d \in \mathbb{N}} \sum_{x=1}^{d-1}(F(0, n / d, x, d)+F(n / d, 0, d, x)+F(n / d, n / d, d-x,-x) \\
- & F(x, x-d, n / d, n / d)-F(x, d, 0, n / d)-F(d, x, n / d, 0))
\end{aligned}
$$

Taking $F(a, b, x, y)=f(b)$ in Theorem 3.1, where $f: \mathbb{Z} \rightarrow \mathbb{C}$ is an even function, we obtain

Corollary 3.1. Let $f: \mathbb{Z} \rightarrow \mathbb{C}$ be an even function. Then for $n \in \mathbb{N}$ we have

$$
\begin{aligned}
& \sum_{\substack{(a, b, x, y) \in \mathbb{N}^{4} \\
a x+b y=n}}(f(a-b)-f(a+b)) \\
= & f(0)(\sigma(n)-d(n))+\sum_{d \mid n} f(d)-\sum_{d \mid n} d f(d)+2 \sum_{d \mid n} \frac{n}{d} f(d)-2 \sum_{d \mid n} \sum_{1 \leq l \leq d} f(l) .
\end{aligned}
$$

Corollary 3.1 was stated but not proved by Liouville in [9]. Replacing $n$ by $2 n$ in Theorem 3.1, and choosing $F(a, b, x, y)=F_{2}(a) f(b) F_{2}(y)$, where $f: \mathbb{Z}^{4} \rightarrow \mathbb{C}$ is even, we obtain

Corollary 3.2. Let $f: \mathbb{Z} \rightarrow \mathbb{C}$ be an even function. Then for $n \in \mathbb{N}$ we have

$$
\begin{aligned}
& \sum_{\substack{(a, b, x, y) \in \mathbb{N}^{4} \\
a x+b y=n}}(f(2 a-b)-f(2 a+b)) \\
&= f(0)\left(\frac{1}{2} \sigma(n)-\frac{1}{2} d(n)-\frac{1}{2} d\left(\frac{n}{2}\right)\right) \\
&+\frac{1}{2} \sum_{d \mid n} f(d)-\frac{1}{2} \sum_{d \mid n} d f(d)+2 \sum_{d \mid n} \frac{n}{d} f(d) \\
&+\frac{1}{2} \sum_{d \mid n} f(2 d)+\frac{1}{2} \sum_{d \mid n} \frac{n}{d} f(2 d)-\sum_{d \mid n} \sum_{1 \leq l \leq 2 d} f(l)
\end{aligned}
$$




$$
-\sum_{d \mid n} \sum_{\substack{1 \leq l \leq d \\ l \equiv d(\bmod 2)}} f(l)
$$

Let $k \in \mathbb{N}$. Taking $f(x)=F_{k}(x)(x \in \mathbb{Z})$ in Corollary 3.1 and appealing to (2.3), (2.4), (2.5) and (2.6), we obtain

Theorem 3.2. Let $k, n \in \mathbb{N}$. Then

$$
\begin{aligned}
& \sum_{\substack{(a, b, x, y) \in \mathbb{N}^{4} \\
a x+b y=n}}\left(F_{k}(a-b)-F_{k}(a+b)\right) \\
& \quad=\sigma(n)-(k-2) \sigma\left(\frac{n}{k}\right)-d(n)+d\left(\frac{n}{k}\right)-2 \sum_{d \mid n}\left[\frac{d}{k}\right] .
\end{aligned}
$$

Finally, taking $f(x)=F_{k}(x)(x \in \mathbb{Z})$ in Corollary 3.2, and appealing to (2.2), (2.3), (2.4), (2.5), (2.6) and (2.13), we obtain

Theorem 3.3. Let $k, n \in \mathbb{N}$. Then if $k$ is odd we have

$$
\begin{aligned}
& \sum_{\substack{(a, b, x, y) \in \mathbb{N}^{4} \\
a x+b y=n}}\left(F_{k}(2 a-b)-F_{k}(2 a+b)\right) \\
= & \frac{1}{2} \sigma(n)+\frac{(5-k)}{2} \sigma\left(\frac{n}{k}\right)-\frac{1}{2} d(n)-\frac{1}{2} d\left(\frac{n}{2}\right)+d\left(\frac{n}{k}\right) \\
& -\sum_{d \mid n}\left[\frac{2 d}{k}\right]-\sum_{d \mid n / 2}\left[\frac{d}{k}\right]-\sum_{d \mid n}\left[\frac{d+k}{2 k}\right]+\sum_{d \mid n / 2}\left[\frac{2 d+k}{2 k}\right]
\end{aligned}
$$

and if $k$ is even

$$
\begin{aligned}
& \sum_{\substack{(a, b, x, y) \in \mathbb{N}^{4} \\
a x+b y=n}}\left(F_{k}(2 a-b)-F_{k}(2 a+b)\right) \\
& =\frac{1}{2} \sigma(n)+\frac{(4-k)}{2} \sigma\left(\frac{n}{k}\right)+\frac{1}{2} \sigma\left(\frac{n}{k / 2}\right)-\frac{1}{2} d(n)-\frac{1}{2} d\left(\frac{n}{2}\right)+\frac{1}{2} d\left(\frac{n}{k}\right) \\
& \quad+\frac{1}{2} d\left(\frac{n}{k / 2}\right)-\sum_{d \mid n}\left[\frac{2 d}{k}\right]-\sum_{d \mid n / 2}\left[\frac{2 d}{k}\right] .
\end{aligned}
$$

4. Evaluation of some finite sums. Our task in this section is to give the values of the sums $\sum_{d \mid n}\left[\frac{d}{k}\right], \sum_{d \mid n}\left[\frac{2 d}{k}\right], \sum_{d \mid n / 2}\left[\frac{d}{k}\right], \sum_{d \mid n}\left[\frac{d+k}{2 k}\right]$ and $\sum_{d \mid n / 2}\left[\frac{2 d+k}{2 k}\right]$ occurring in Theorems 3.2 and 3.3 in the special case where $k=7$. 
For $a \in \mathbb{Z}$ and $m, n \in \mathbb{N}$ we define

$$
d_{a, m}(n):=\sum_{\substack{d \mid n \\ d \equiv a(\bmod \mathrm{m})}} 1,
$$

so that

$$
\sum_{a=0}^{m-1} d_{a, m}(n)=d(n)
$$

In particular we set

$$
d_{i}:=d_{i, 7}(n), \quad i=0,1,2,3,4,5,6
$$

and

$$
e_{i}:=d_{i, 14}(n), \quad i=0,1,2,3,4,5,6,7,8,9,10,11,12,13 .
$$

Clearly,

$$
d_{i}=e_{i}+e_{i+7}, \quad i=0,1,2,3,4,5,6 .
$$

Also,

$$
d_{0}=d_{0,7}(n)=\sum_{\substack{d \mid n \\ d \equiv 0(\bmod 7)}} 1=\sum_{d \mid n / 7} 1=d\left(\frac{n}{7}\right)
$$

and, similarly,

$$
e_{0}=d\left(\frac{n}{14}\right)
$$

Thus

$$
e_{7}=d_{0}-e_{0}=d\left(\frac{n}{7}\right)-d\left(\frac{n}{14}\right) .
$$

We need the following results, all of which are simple to prove.

$$
\begin{aligned}
d(n)= & e_{0}+e_{1}+e_{2}+\cdots+e_{13} . \\
d\left(\frac{n}{2}\right)= & e_{0}+e_{2}+e_{4}+\cdots+e_{12} . \\
d\left(\frac{n}{7}\right)= & e_{0}+e_{7} . \\
d_{i, 7}\left(\frac{n}{2}\right)= & e_{2 i}, \quad i=0,1,2, \ldots, 6 . \\
\sum_{d \mid n}\left[\frac{d}{7}\right]= & \frac{1}{7} \sigma(n)-\frac{1}{7}\left(e_{1}+2 e_{2}+3 e_{3}+4 e_{4}+5 e_{5}+6 e_{6}\right. \\
& \left.+e_{8}+2 e_{9}+3 e_{10}+4 e_{11}+5 e_{12}+6 e_{13}\right) . \\
\sum_{d \mid n}\left[\frac{2 d}{7}\right]= & \frac{2}{7} \sigma(n)-\frac{1}{7}\left(2 e_{1}+4 e_{2}+6 e_{3}+e_{4}+3 e_{5}+5 e_{6}\right. \\
& \left.+2 e_{8}+4 e_{9}+6 e_{10}+e_{11}+3 e_{12}+5 e_{13}\right) .
\end{aligned}
$$




$$
\begin{aligned}
\sum_{d \mid n}\left[\frac{d+7}{14}\right]= & \frac{1}{14} \sigma(n)-\frac{1}{14}\left(e_{1}+2 e_{2}+3 e_{3}+4 e_{4}+5 e_{5}+6 e_{6}\right. \\
& \left.-7 e_{7}-6 e_{8}-5 e_{9}-4 e_{10}-3 e_{11}-2 e_{12}-e_{13}\right) . \\
\sum_{d \mid n / 2}\left[\frac{d}{7}\right]= & \frac{1}{7} \sigma\left(\frac{n}{2}\right)-\frac{1}{7}\left(e_{2}+2 e_{4}+3 e_{6}+4 e_{8}+5 e_{10}+6 e_{12}\right) . \\
\sum_{d \mid n / 2}\left[\frac{2 d+7}{14}\right]= & \frac{1}{7} \sigma\left(\frac{n}{2}\right)-\frac{1}{7}\left(e_{2}+2 e_{4}+3 e_{6}-3 e_{8}-2 e_{10}-e_{12}\right) .
\end{aligned}
$$

We are now in a position to prove the three theorems that we will need in the proof of Theorem 1.1 in Section 5.

Theorem 4.1. Let $n \in \mathbb{N}$. Then

$$
\begin{aligned}
& \sum_{\substack{(a, b, x, y) \in \mathbb{N}^{4} \\
a x+b y=n}}\left(F_{7}(a-b)-F_{7}(a+b)\right) \\
& \quad=\frac{5}{7} \sigma(n)-5 \sigma\left(\frac{n}{7}\right)-\frac{5}{7} d_{1}-\frac{3}{7} d_{2}-\frac{1}{7} d_{3}+\frac{1}{7} d_{4}+\frac{3}{7} d_{5}+\frac{5}{7} d_{6} .
\end{aligned}
$$

Proof. This result follows by taking $k=7$ in Theorem 3.2 and appealing to (4.5), (4.9), (4.11) and (4.13).

Theorem 4.2. Let $n \in \mathbb{N}$. Then

$$
\begin{aligned}
& \sum_{\substack{(a, b, x, y) \in \mathbb{N}^{4} \\
a x+b y=n}}\left(F_{7}(2 a-b)-F_{7}(2 a+b)\right) \\
& \quad=\frac{1}{7} \sigma(n)-\sigma\left(\frac{n}{7}\right)-\frac{1}{7} d_{1}-\frac{2}{7} d_{2}+\frac{4}{7} d_{3}-\frac{4}{7} d_{4}+\frac{2}{7} d_{5}+\frac{1}{7} d_{6} .
\end{aligned}
$$

Proof. This result follows by taking $k=7$ in Theorem 3.3 and appealing to (4.5), (4.9), (4.10), (4.11), (4.14), (4.15), (4.16) and (4.17).

Theorem 4.3. For all $a, b \in \mathbb{N}$

$$
\begin{aligned}
\left(\frac{-7}{a b}\right)= & \left(F_{7}(a-b)-F_{7}(a+b)\right)+\left(F_{7}(a-2 b)-F_{7}(a+2 b)\right) \\
& +\left(F_{7}(2 a-b)-F_{7}(2 a+b)\right) .
\end{aligned}
$$

Proof. If $a \equiv 0(\bmod 7)$ or $b \equiv 0(\bmod 7)$ both the left-hand side and right-hand side of the asserted formula are zero. Thus we may suppose that $a \not \equiv 0(\bmod 7)$ and $b \not \equiv 0(\bmod 7)$. Define $c \not \equiv 0(\bmod 7)$ by $a \equiv b c(\bmod 7)$. Then the assertion of the theorem becomes

$$
\left(\frac{-7}{c}\right)=\left(F_{7}(c-1)-F_{7}(c+1)\right)+\left(F_{7}(c-2)-F_{7}(c+2)\right)
$$




$$
+\left(F_{7}(2 c-1)-F_{7}(2 c+1)\right) .
$$

This is easily checked for the six cases $c \equiv 1,2,3,4,5,6(\bmod 7)$.

5. Proof of Theorem 1.1. For $m \in \mathbb{N}_{0}$ we let

$$
r(m)=\text { number of }(x, y) \in \mathbb{Z}^{2} \text { such that } x^{2}+x y+2 y^{2}=m \text {. }
$$

Clearly,

$$
r(0)=1 \text {. }
$$

For $n \in \mathbb{N}$ it is a classical result that

$$
r(n)=2 \sum_{d \mid n}\left(\frac{-7}{d}\right) .
$$

Thus

$$
r(n)=2 d_{1}+2 d_{2}-2 d_{3}+2 d_{4}-2 d_{5}-2 d_{6} .
$$

The number of $(x, y, z, t) \in \mathbb{Z}^{4}$ such that

$$
n=x^{2}+x y+2 y^{2}+z^{2}+z t+2 t^{2}
$$

is (appealing to (5.2), (5.3), Theorem 4.3, Theorem 4.1, Theorem 4.2 and (5.4))

$$
\begin{aligned}
& \sum_{\substack{(k, l) \in \mathbb{N}_{0}^{2} \\
k+l=n}} r(k) r(l)=2 r(n)+\sum_{k=1}^{n-1} r(k) r(n-k) \\
& =2 r(n)+\sum_{k=1}^{n-1}\left(2 \sum_{a \mid k}\left(\frac{-7}{a}\right)\right)\left(2 \sum_{b \mid n-k}\left(\frac{-7}{b}\right)\right) \\
& =2 r(n)+4 \sum_{\substack{(a, b, x, y) \in \mathbb{N}^{4} \\
a x+b y=n}}\left(\frac{-7}{a b}\right) \\
& =2 r(n)+4 \sum_{\substack{(a, b, x, y) \in \mathbb{N}^{4} \\
a x+b y=n}}\left(F_{7}(a-b)-F_{7}(a+b)\right) \\
& +4 \sum_{\substack{(a, b, x, y) \in \mathbb{N}^{4} \\
a x+b y=n}}\left(F_{7}(a-2 b)-F_{7}(a+2 b)\right) \\
& +4 \sum_{\substack{(a, b, x, y) \in \mathbb{N}^{4} \\
a x+b y=n}}\left(F_{7}(2 a-b)-F_{7}(2 a+b)\right) \\
& =2 r(n)+4 \sum_{\substack{(a, b, x, y) \in \mathbb{N}^{4} \\
a x+b y=n}}\left(F_{7}(a-b)-F_{7}(a+b)\right)
\end{aligned}
$$




$$
\begin{gathered}
+8 \sum_{\substack{(a, b, x, y) \in \mathbb{N}^{4} \\
a x+b y=n}}\left(F_{7}(2 a-b)-F_{7}(2 a+b)\right) \\
=2 r(n)+4\left(\frac{5}{7} \sigma(n)-5 \sigma\left(\frac{n}{7}\right)-\frac{5}{7} d_{1}-\frac{3}{7} d_{2}-\frac{1}{7} d_{3}+\frac{1}{7} d_{4}+\frac{3}{7} d_{5}+\frac{5}{7} d_{6}\right) \\
\quad+8\left(\frac{1}{7} \sigma(n)-\sigma\left(\frac{n}{7}\right)-\frac{1}{7} d_{1}-\frac{2}{7} d_{2}+\frac{4}{7} d_{3}-\frac{4}{7} d_{4}+\frac{2}{7} d_{5}+\frac{1}{7} d_{6}\right) \\
=2 r(n)+4 \sigma(n)-28 \sigma\left(\frac{n}{7}\right)-4 d_{1}-4 d_{2}+4 d_{3}-4 d_{4}+4 d_{5}+4 d_{6} \\
=4 \sigma(n)-28 \sigma\left(\frac{n}{7}\right) .
\end{gathered}
$$

This completes the proof of Theorem 1.1.

6. Proof of Theorem 1.2. We have by (1.5), Theorem 1.1 and (1.4)

$$
\begin{aligned}
S^{2}(q) & =\sum_{x, y, z, t \in \mathbb{Z}} q^{x^{2}+x y+2 y^{2}+z^{2}+z t+2 t^{2}} \\
& =1+\sum_{n=1}^{\infty}\left(4 \sigma(n)-28 \sigma\left(\frac{n}{7}\right)\right) q^{n} \\
& =\frac{7}{6} L\left(q^{7}\right)-\frac{1}{6} L(q) .
\end{aligned}
$$

7. Proof of Theorem 1.3. Let $n \in \mathbb{N}$. Set

$$
A(n):=\left\{(x, y, z, t) \in \mathbb{Z}^{4} \mid 4 n=x^{2}+y^{2}+7 z^{2}+7 t^{2}, x \equiv z(\bmod 2)\right\}
$$

and

$$
B(n):=\left\{(x, y, z, t) \in \mathbb{Z}^{4} \mid n=x^{2}+x y+2 y^{2}+z^{2}+z t+2 t^{2}\right\} .
$$

Let $(x, y, z, t) \in A(n)$. Then $4 n=x^{2}+y^{2}+7 z^{2}+7 t^{2}$ and $x \equiv z(\bmod 2)$ so $\frac{x-z}{2} \in \mathbb{Z}$ and

$$
y-t \equiv y^{2}-t^{2} \equiv y^{2}+7 t^{2}=4 n-x^{2}-7 z^{2} \equiv x-z \equiv 0(\bmod 2)
$$

so $\frac{y-t}{2} \in \mathbb{Z}$. Further

$$
\begin{gathered}
\left(\frac{x-z}{2}\right)^{2}+\left(\frac{x-z}{2}\right) z+2 z^{2}+\left(\frac{y-t}{2}\right)^{2}+\left(\frac{y-t}{2}\right) t+2 t^{2} \\
=\frac{1}{4}\left(x^{2}+7 z^{2}+y^{2}+7 t^{2}\right)=n
\end{gathered}
$$

so $\left(\frac{x-z}{2}, z, \frac{y-t}{2}, t\right) \in B(n)$. Thus we can define $\lambda: A(n) \rightarrow B(n)$ by

$$
\lambda((x, y, z, t))=\left(\frac{x-z}{2}, z, \frac{y-t}{2}, t\right) .
$$


Clearly, $\lambda$ is injective. Let $\left(x_{1}, y_{1}, z_{1}, t_{1}\right) \in B(n)$. Set $x=2 x_{1}+y_{1} \in \mathbb{Z}$, $y=2 z_{1}+t_{1} \in \mathbb{Z}, z=y_{1} \in \mathbb{Z}, t=t_{1} \in \mathbb{Z}$. Clearly, $x \equiv y_{1} \equiv z(\bmod 2)$. Also

$$
\begin{aligned}
x^{2}+y^{2}+7 z^{2}+7 t^{2} & =\left(2 x_{1}+y_{1}\right)^{2}+\left(2 z_{1}+t_{1}\right)^{2}+7 y_{1}^{2}+7 t_{1}^{2} \\
& =4\left(x_{1}^{2}+x_{1} y_{1}+2 y_{1}^{2}+z_{1}^{2}+z_{1} t_{1}+2 t_{1}^{2}\right)=4 n .
\end{aligned}
$$

Hence $(x, y, z, t) \in A(n)$. Moreover

$$
\lambda((x, y, z, t))=\left(\frac{x-z}{2}, z, \frac{y-t}{2}, t\right)=\left(x_{1}, y_{1}, z_{1}, t_{1}\right)
$$

so $\lambda$ is surjective. Thus $\lambda$ is a bijection and we have by Theorem 1.1

$$
\text { card } A(n)=\operatorname{card} B(n)=4 \sigma(n)-28 \sigma\left(\frac{n}{7}\right)=4 \sum_{\substack{d \mid n \\ 7 \nmid d}} d
$$

as asserted.

8. Proof of Theorem 1.4. Let $n \in \mathbb{N}$. Let $N(n)$ denote the number of $\left(x_{1}, x_{2}, x_{3}, x_{4}, x_{5}, x_{6}, x_{7}, x_{8}\right) \in \mathbb{Z}^{8}$ such that

$$
n=x_{1}^{2}+x_{1} x_{2}+2 x_{2}^{2}+x_{3}^{2}+x_{3} x_{4}+2 x_{4}^{2}+x_{5}^{2}+x_{5} x_{6}+2 x_{6}^{2}+x_{7}^{2}+x_{7} x_{8}+2 x_{8}^{2} \text {. }
$$

Then by Theorem 1.2 we have

$$
\sum_{n=0}^{\infty} N(n) q^{n}=\left(\sum_{(x, y) \in \mathbb{Z}^{2}} q^{x^{2}+x y+2 y^{2}}\right)^{4}=S^{4}(q)=\frac{1}{36}\left(L(q)-7 L\left(q^{7}\right)\right)^{2} .
$$

Appealing to [8, Lemma 4.6, p. 113] we obtain

$$
\sum_{n=0}^{\infty} N(n) q^{n}=1+\sum_{n=1}^{\infty}\left(\frac{24}{5} \sigma_{3}(n)+\frac{1176}{5} \sigma_{3}\left(\frac{n}{7}\right)+\frac{16}{5} c_{7}(n)\right) q^{n} .
$$

Equating coefficients of $q^{n}(n \in \mathbb{N})$, we obtain the asserted result.

9. Proof of Theorem 1.5. As in [8, equation (4.1), p. 112] we define

$$
H=\left(\frac{A^{7}}{C}+13 q A^{3} C^{3}+49 q^{2} \frac{C^{7}}{A}\right)^{\frac{1}{3}}
$$

where

$$
A:=\prod_{n=1}^{\infty}\left(1-q^{n}\right), \quad C:=\prod_{n=1}^{\infty}\left(1-q^{7 n}\right) .
$$

From the proof of Theorem 1.4 and [8, Lemma 4.2, p. 112] we have

$$
S^{4}(q)=\frac{1}{36}\left(L(q)-7 L\left(q^{7}\right)\right)^{2}=H^{4}
$$

so that $S(q)=\omega(q) H(q)$, where $\omega(q)^{4}=1$. From (1.5) and (9.1) we find for $|q|<1$ that $S(q)=1+2 q+4 q^{2}+O\left(q^{3}\right)$ and $H=1+2 q+4 q^{2}+O\left(q^{3}\right)$ so that $\omega(q)=1$ and

$$
H=S(q)
$$


This also follows from [2, Lemma 2.2, p. 1737] (with a typo corrected). Next, by $[8$, Lemma 4.4 , p. 112] (with a typo corrected ) and (9.3) we have

$$
\sum_{n=1}^{\infty} c_{7}(n) q^{n}=q A^{3} C^{3} H=q A^{3} C^{3} S(q) .
$$

Now, by (1.5), (5.1), (5.2) and (5.3), we have

$$
S(q)=\sum_{x, y=-\infty}^{\infty} q^{x^{2}+x y+2 y^{2}}=\sum_{n=0}^{\infty} r(n) q^{n}=1+2 \sum_{n=1}^{\infty} \sum_{d \mid n}\left(\frac{-7}{d}\right) q^{n} .
$$

Hence, from (9.2), (9.4) and (9.5), we deduce

$$
\begin{aligned}
\sum_{n=1}^{\infty} c_{7}(n) q^{n}=q \prod_{n=1}^{\infty}( & \left.1-q^{n}\right)^{3} \prod_{n=1}^{\infty}\left(1-q^{7 n}\right)^{3} \\
& \times\left(1+2 \sum_{n=1}^{\infty} \sum_{d \mid n}\left(\frac{-7}{d}\right) q^{n}\right) .
\end{aligned}
$$

By Jacobi's identity [7, Corollary 6, p. 37]

$$
\prod_{n=1}^{\infty}\left(1-q^{n}\right)^{3}=\sum_{r=0}^{\infty}(-1)^{r}(2 r+1) q^{\frac{r(r+1)}{2}}
$$

equation (9.6) becomes

$$
\begin{aligned}
& \sum_{n=1}^{\infty} c_{7}(n) q^{n-1}=\left(\sum_{r=0}^{\infty}(-1)^{r}(2 r+1) q^{\frac{r(r+1)}{2}}\right)\left(\sum_{s=0}^{\infty}(-1)^{s}(2 s+1) q^{\frac{7 s(s+1)}{2}}\right) \\
& +2\left(\sum_{r=0}^{\infty}(-1)^{r}(2 r+1) q^{\frac{r(r+1)}{2}}\right)\left(\sum_{s=0}^{\infty}(-1)^{s}(2 s+1) q^{\frac{7 s(s+1)}{2}}\right)\left(\sum_{t=1}^{\infty} \sum_{d \mid t}\left(\frac{-7}{d}\right) q^{t}\right) \\
& =\sum_{n=1}^{\infty}\left(\sum_{r, s=0}^{\infty}(-1)^{r+s}(2 r+1)(2 s+1)\right) q^{n-1} \\
& +2 \sum_{n=1}^{\infty}\left(\sum_{r, s=0}^{\frac{r(r+1)}{2}+\frac{7 s(s+1)}{2}=n-1} \sum_{t=1}^{\infty}(-1)^{r+s}(2 r+1)(2 s+1) \sum_{d \mid t}\left(\frac{-7}{d}\right)\right) q^{n-1} .
\end{aligned}
$$

Equating coefficients of $q^{n-1}(n \in \mathbb{N})$ we obtain the asserted formula for $c_{7}(n)$.

\section{ACKNOWLEDGEMENTS}

The author's research was supported by Natural Sciences and Engineering Research Council of Canada grant A-7233. The author thanks Matt Lemire who checked Theorem 1.5 numerically for $n=1, \ldots, 100$. 


\section{REFERENCES}

1. B. C. Berndt, Ramanujan's notebooks. Part III. Springer-Verlag, New York, 1991.

2. H. H. Chan and Y. L. Ong, On Eisenstein series and $\sum_{m, n=-\infty}^{\infty} q^{m^{2}+m n+2 n^{2}}$. Proc. Amer. Math. Soc. 127(1999), No. 6, 1735-1744.

3. J. G. Huard, Z. M. Ou, B. K. Spearman, and K. S. Williams, Elementary evaluation of certain convolution sums involving divisor functions. Number theory for the millennium, II (Urbana, IL, 2000), 229-274, A K Peters, Natick, MA, 2002.

4. G. Humbert, Sur la mesure de l'ensemble des classes positive d'Hermite, de discriminant donné, dans un corps quadratique imaginaire. Comptes Rendus Paris 169(1919), 407-414.

5. N. KaChaKhidze, On the representation of numbers by a direct sum of quadratic forms $x_{1}^{2}+x_{1} x_{2}+2 x_{2}^{2}$. Soobshch. Akad. Nauk. Gruzii 146(1992), No. 3, 474-477.

6. F. KLeIn and R. Fricke, Elliptischen Modulfunctionen, vol. 2, 1892.

7. M. I. KNopp, Modular functions in analytic number theory. 2nd ed. Chelsea Publ. Co., New York, 1993.

8. M. F. Lemire and K. S. Williams, Evaluation of two convolution sums involving the sum of divisors function. Bull. Austral. Math. Soc. 73(2006), 107-115.

9. J. Liouville, Sur quelques formules générales qui peuvent être utiles dans la théorie des nombres (fifth article). J. Math. Pures Appl. 3(1858), 273-288.

10. S. Ramanujan, Notebooks. Vols. 1, 2. Tata Institute of Fundamental Research, Bombay, 1957.

(Received 9.08.2006)

Author's address:

Centre for Research in Algebra and Number Theory

School of Mathematics and Statistics

Carleton University

Ottawa, Ontario K1S 5B6

Canada

E-mail: kwilliam@connect.carleton.ca 